\title{
Electrode surface modified with heteropoly or isopoly anions: STM and AFM characterization
}

\author{
L. NADJO and B. KEITA
}

\author{
Laboratoire d'Electrochimie et de Photoélectrochimie, URA 1383 du CNRS, Université de Paris XI, \\ Bâtiment 420, 91405 Orsay cedex, France
}

Abstract :

It has been shown on two examples that STM and AFM techniques can be used to image oxometalates with molecular resolution. The shapes of these molecules are clearly distinguished and their dimensions have been determined. It has even been possible to correlate other results culled from the AFM image of the surface of a single crystal of an oxometalate with those of crystallography. These results served as reference images in the study of the basal plane of HOPG when it is used as a working electrode during the cyclic voltammetry of $10^{-3} \mathrm{M} \mathrm{H}_{2} \mathrm{~W}_{12} \mathrm{O}_{40}^{-}$, in a $\mathrm{pH}=2$ medium, with simultaneous STM monitoring of the surface morphology. A potential-dependent deposition of the oxometalate is superimposed on a strong alteration of the HOPG surface. When the potential domain is extended to include the third redox system of $\mathrm{H}_{2} \mathrm{~W}_{12} \mathrm{O}_{40}^{6-}$, a particularly well-ordered two-dimensional array is observed, the individual entities having the diameter of the oxometalate.

Recently, the catalytic properties of electrode surfaces modified with heteropoly or isopolyanions have been described in several papers from this laboratory [1-4]. Two types of modifications were studied. Materials as diverse as glassy carbon, graphite, gold, tungsten, etc, or semiconductor like p-type Si have been modified by direct electrodeposition of oxometalate-based catalysts. Also, it has been possible to include these oxometalates in organic polymers or in clays, previously deposited on electrode surfaces. Whatever the technique, the observed catalytic properties must be undoubtedly by traced to the presence of the oxometalates or to their reduction products. Therefore, these species which appear as molecular soluble analogs of metal oxides or metal oxide mixtures, offer a rare opportunity to study chemical and structural transformations of the catalysts on well-defined species. As a matter of fact, little is known about the geometrical factors within the molecules, or their spatial distribution or chemical transformations which, all together, could induce the observed catalytic properties. Then, nearfield microscopies, many of which can be operated in-situ during electrochemical experiments, appear as very appropriate tools to try and monitor topographical changes related to volume properties in catalysts at work. Among the necessary steps towards this end, it must be proved that heteropoly and isopolyanions can be imaged with a high spatial resolution. The first part of this paper is therefore devoted to the STM and AFM study of this class of compounds [5-8]. The second part discusses some results obtained when electrode surfaces are imaged in-situ at a molecular or even an atomic resolution during the scanning of the cyclic voltammogram of an oxometalate [9].

A Nanoscope II, operating in air and at room temperature was used throughout (Digital Instruments, Santa Barbara, CA). Properly implemented, the same apparatus is used for AFM experiments. The Nanoscope II can also be equipped with a bipotentiostat for simultaneous electrochemical measurements and STM or AFM imaging (ECSTM or ECAFM). For ECSTM, W or $\mathrm{Pt} / \mathrm{Ir}(80 / 20)$ tips are coated with Apiezon was, except for their very end. To further minimize the faradic current through the tunneling tip, the tip/surface bias voltage was usually fixed so that parallel variations occur in the tip/electrode potential during voltammetric runs. 
Ex-situ STM and AFM studies [5, 6, 8]

$\mathrm{PW}_{12} \mathrm{O}_{40}^{3-}$ which is one of the precursors used for electrode modications [1-4] has been selected as a first example. Like most transition metal polyoxoanions, it is constituted by discrete molecules, with their structures based on close-packed oxygen arrays containing interstitial metal centers. This very symmetrical structure is easily accommodated in a sphere of $11 \AA$ diameter. A drop of methanolic solution of $\mathrm{H}_{3} \mathrm{PW}_{12} \mathrm{O}_{40}$ (typically $10^{-4} \mathrm{M}$ ) was deposited on the freshly-cleaved surface of a piece of highly oriented pyrolytic graphite (HOPG) and the solvent was allowed to evaporate. Figure 1 shows current-mode STM images of this surface. The observed structure is stable, well-ordered in several areas, with parameters clearly different from those of the underlying HOPG. In the constant currentmode STM, the tunnel tip traces out contours of constant density of states and thus, probes the topology of the electron distribution above the surface of the sample. Therefore, the structures in figure 1 , which closely resemble what could be expected from the crystallographic shape of $\mathrm{PW}_{12} \mathrm{O}_{40}^{3-}$, suggest that individual oxometalate species are imaged. The mean diameter, deduced from numerous measurements [5] is $9.6 \pm 0.4 \AA$, which is very close to the crystallographic diameter.

With the aim of further demonstrating the ability of STM and AFM techniques to show the shape of oxometalates and to help in their size determinations, sodiumdecatungstocerate (IV)wasselected as a new representative example. This molecule $\mathrm{Na}_{6} \mathrm{H}_{2} \mathrm{CeW}_{10} \mathrm{O}_{36} \cdot 30 \mathrm{H}_{2} \mathrm{O}$ is rather ellipsoïdal in shape. STM studies on this compound deposited on HOPG and AFM imaging on a crystal wet with solvent give very similar results, from which the molecular dimensions could be measured. Other conclusions could be drawn as well $[6,8]: \mathrm{i}$ ) in the case of oxometalates, a substrate like HOPG has no detectable influence on the STM image. ii) the STM image corresponds to a purely topographic representation of the surface. iii) for oxometalates, the correlation between images obtained by the two techniques is excellent. These conclusions have received confirmation through the AFM and crystallographic studies of a single crystal of sodium decatungstocerate (IV). Figure 2 shows the AFM image of the surface of such a "dry" crystal. Even though slightly "faulted" areas exist, the pattern is largely dominated by a periodic arrangement of molecules. The molecular shape is easily observed, and the molecular dimensions have been measured with a fair accuracy. The very regularity of the observed pattern prompted us to try and fit the AFM images with the crystallographic arrangements of the molecules in the single crystal. Sodium decatungstocerate (IV) crystallizes with 30 water molecules and belongs to the monoclinic system, space group $\mathrm{C}_{2} / \mathrm{c}, \mathrm{a}=18.14 \AA, \mathrm{b}=18.62$ $\AA, c=18.51 \AA, \beta=95.9^{\circ}$ and $Z=4$. We have generated a computer representation of the arrangement of the heteropolyanion in a projection along the b-axis. This arrangement has been chosen because it seems to correspond best to the AFM image. The fitting of this computer arrangement to a zoomed AFM pattern shows that the angles in the apparent unit-cell are slightly
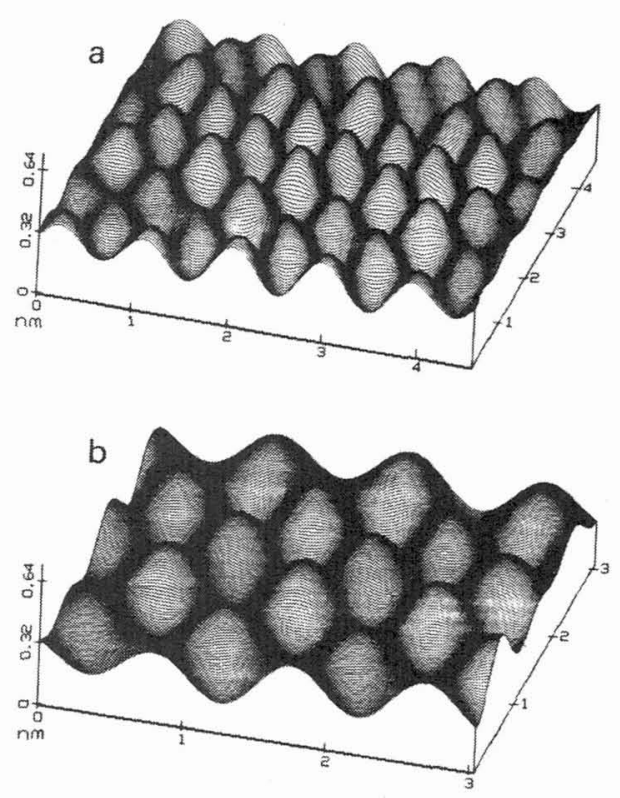

Fig. 1. STM images of $\mathrm{H}_{3} \mathrm{PW}_{12} \mathrm{O}_{40}$ in air at $298 \mathrm{~K}(J=1.1 \mathrm{nA}$, $U=201.4 \mathrm{mV}$ ). Images have been filtered by a two-dimensional fast-Fourier-transform. Pitch angle: $30^{\circ}$. (a) Three dimensional line-plot image. (b) Zoomed three dimensional lineplot image. 
Also, the experimental distances deduced from the AFM image appear toapproach crystallographic determinations to within less than $5 \%$. All these results together suggest that the scanned area represents actually the $(010)$ face of the crystal and appears as a simple termination of the bulk.

\section{Simultaneous STM imaging and cyclic voltammetry [9]}

The conclusion from the aforementioned results is that fairly adherent deposits of the oxometalates on HOPG can be imaged by STM or AFM, showing recognizably the shapes of the molecules and allowing for determination of molecular dimensions. Starting with such reference images, an electrochemical study with simultaneous STM imaging has been undertaken with a solution containing $10^{-3} \mathrm{M}\left(\mathrm{NH}_{4}\right)_{6} \mathrm{H}_{2} \mathrm{~W}_{12} \mathrm{O}_{40}$, in a $\mathrm{pH}=2$ medium. The electrochemical cell was the one received with the Nanoscope, with two $\mathrm{Pt}$ wires as counter and reference electrodes respectively. The working electrode is the basal plane of a freshly etched HOPG of $0.41 \mathrm{~cm}^{2}$ surface area. It has been checked that the $\mathrm{Pt}$ wire acts as a quasi-reference electrode : as a matter of fact, the present oxoanion is known to generate stable reduction products upon its first several electronations, and to constitute nernstian systems with them. This assumption is reinforced by the following observation : the cathodic peak potential difference between the first two reduction waves of $\mathrm{H}_{2} \mathrm{~W}_{12} \mathrm{O}_{40}^{6-}$, measured with the present electrochemical set-up, is and remains to less than $10 \mathrm{mV}$ the same as obtained in a conventional set-up with a saturated calomel electrode as the reference. Figure 3 shows a reference cyclic voltammogram of $\mathrm{H}_{2} \mathrm{~W}_{12} \mathrm{O}_{40}^{6-}$ in a $\mathrm{pH}=2$ medium, restricted to the first three waves and runned in a conventional electrochemical cell. The first two waves are bielectronic, the third one corresponds to approximately ten electrons $[10,11]$. All the other cyclic voltammograms have been obtained in the ECSTM cell.

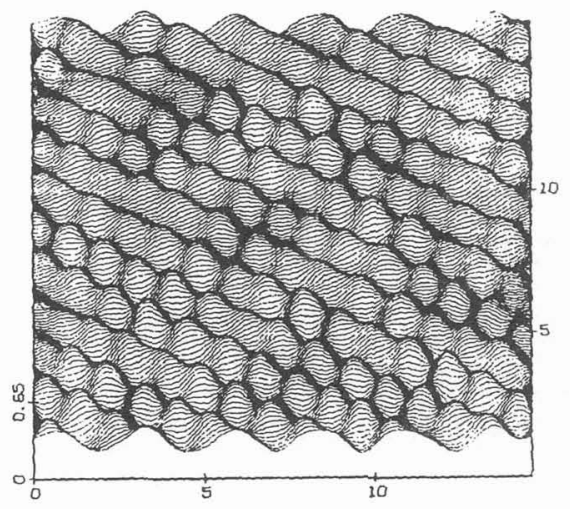

Figure 2:

Line-plot AFM image of the surface of sodium decatungstocerate (IV) in air at room temperature. The image has been filtered by twodimensional fast Fourier-transform.

Figure 4 shows a current-mode STM image of the atomic structure of the HOPG surface in the presence of the $10^{-3} \mathrm{M} \mathrm{H}_{2} \mathrm{~W}_{12} \mathrm{O}_{40}^{6-} \mathrm{pH}=2$ solution, and without any potential applied through the cell. This image is identical with that obtained in air in the absence of liquid. No filtering has been applied. Although similar results have been obtained previously in different electrolytes and even with the sample under potential control [12-14], it is worth noticing the very good resolution of the image of figure 4 , which proves the excellent quality of the coated tips used in the present work. 


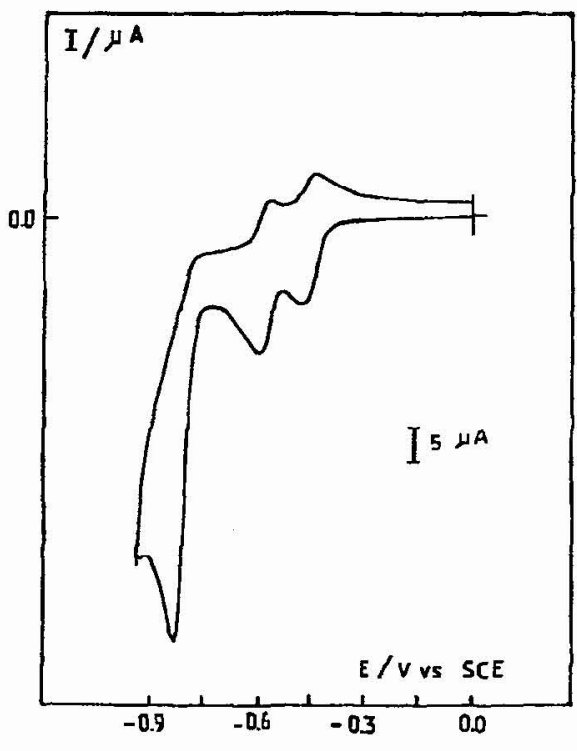

Figure 3

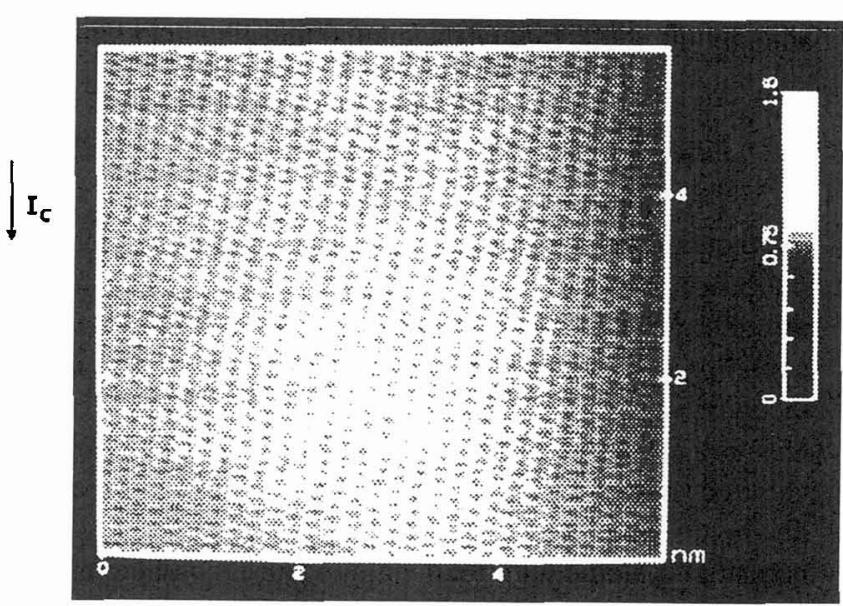

Figure 4

When the potential control is set in and the potential cycling restricted to the double layer region up to the very beginning of the first wave, an alteration of the electrode surface appears in the long run. The STM image of figure 5a has not been filtered. It illustrates the alteration of the surface which shows valleys and ridges oriented from top left corner to bottom right corner in the display, with a corrugation about $0.1 \mathrm{~nm}$ to $0.2 \mathrm{~nm}$ between them. A very few randomly distributed spheric entities with a diameter which would fit that of $\mathrm{H}_{2} \mathrm{~W}_{12} \mathrm{O}_{40}^{6-}$, are detected on the surface. However, the presence of perturbed HOPG surface largely dominates, as confirmed by figure $5 \mathrm{~b}$ which has been obtained by zooming in a part of figure $5 \mathrm{a}$ and filtering by fast-Fourier transform.

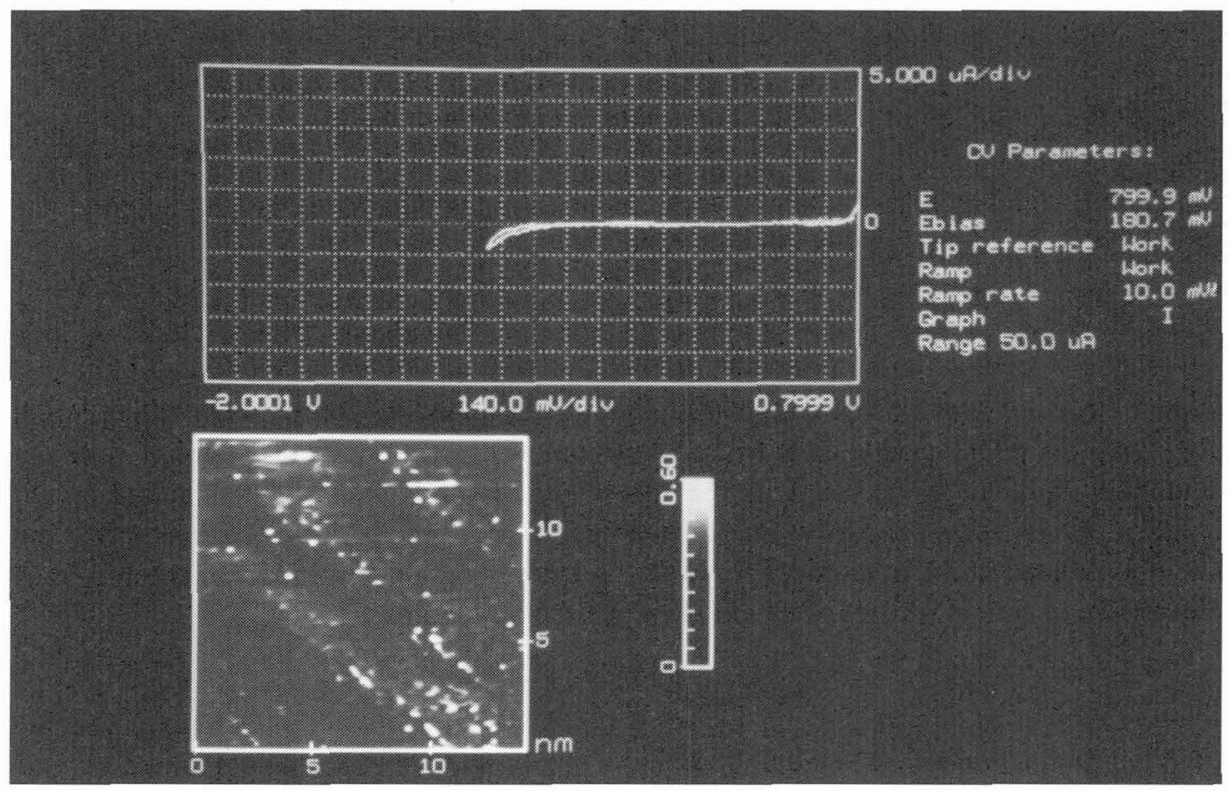

Figure $5 a$ 


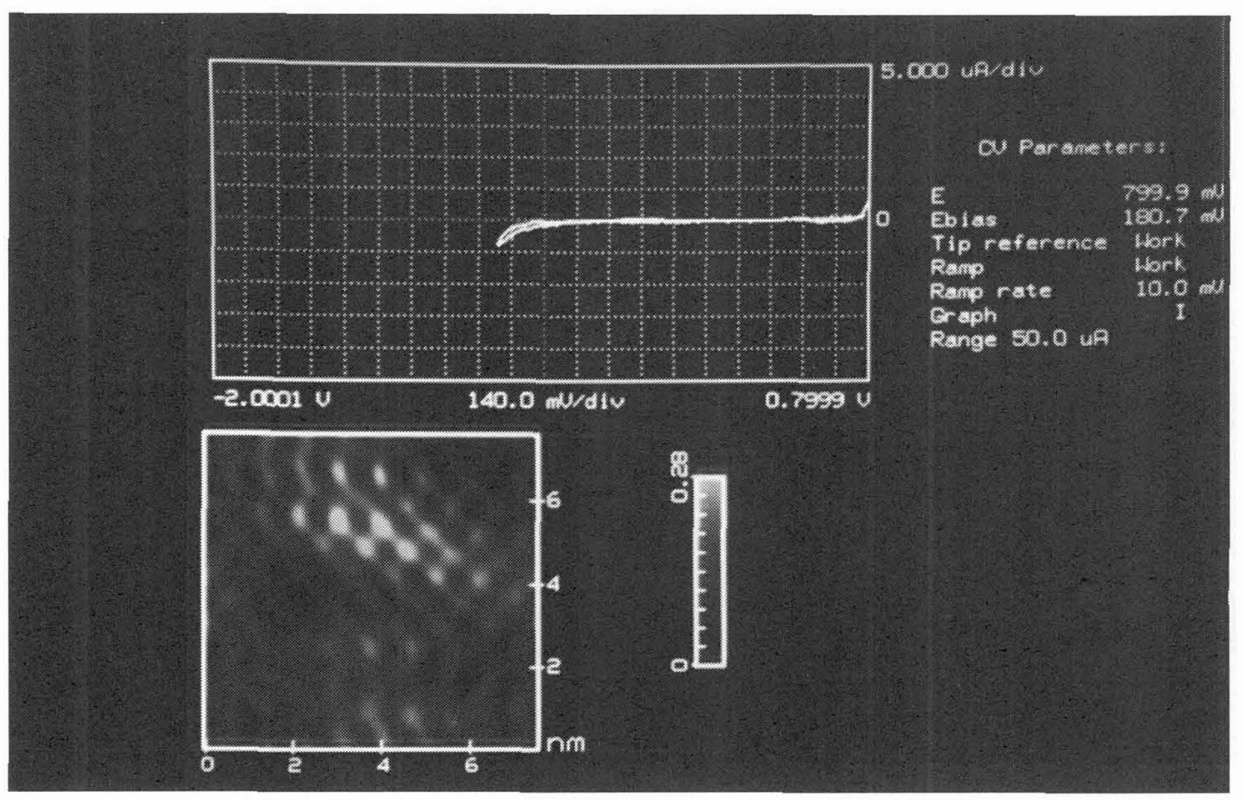

Figure $5 b$

Extension of the potential domain to include the entire first redox system of $\mathrm{H}_{2} \mathrm{~W}_{12} \mathrm{O}_{40}^{6}$ does not change fundamentally the observation as the graphite is still essentially detected. The observed phenomena constitute a smooth transition from the pattern obtained in figure 5 and that obtained in the potential domain of the second redox couple.

The cyclic voltammogram on figure 6a shows no adsorption phenomena, while the STM image reveals a strong perturbation as appears on figure $6 \mathrm{~b}$. This figure has been filtered by fast-Fourier transform. The HOPG surface looks complety amorphous, with a large number of spherical entities with a diameter in the range of $10.5 \pm 0.5 \AA$. Images 5 and 6 constitute a direct illustration of the potential dependence of the adsorptive deposition of $\mathrm{H}_{2} \mathrm{~W}_{12} \mathrm{O}_{40}^{6-}$ and other oxometalates [1-4] on electrode surfaces. They suggest that the deposition process involves primarily formation of islands on the bare surface as long as the layers remain thin enough $[5,15]$. The simultaneous recording of the cyclic voltammogram and of the STM image reveals a remarkable result ; while some adsorption is shown by the STM image, the phenomenon is not still strong enough to distort the cyclic voltammogram, which remains dominated by the solution phase couple.

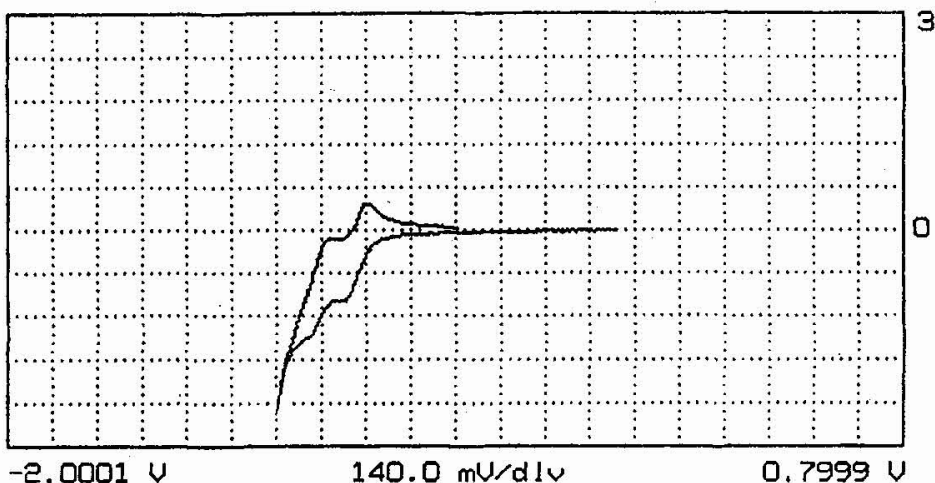

$30.000 \mathrm{uA} / \mathrm{div}$

CU Parameters:

$\begin{array}{lr}\text { E } & 50.0 \mathrm{mU} \\ \text { Etip } & 643.0 \mathrm{mU} \\ \text { Tip reference } & \text { Ref } \\ \text { Wamp } & \text { Work } \\ \text { Ramp rate } & 10.0 \mathrm{mU} / \mathrm{S} \\ \text { Graph } & \mathrm{I} \\ \text { Range } 300.0 \mathrm{uA} & \end{array}$

Figure $6 a$ 


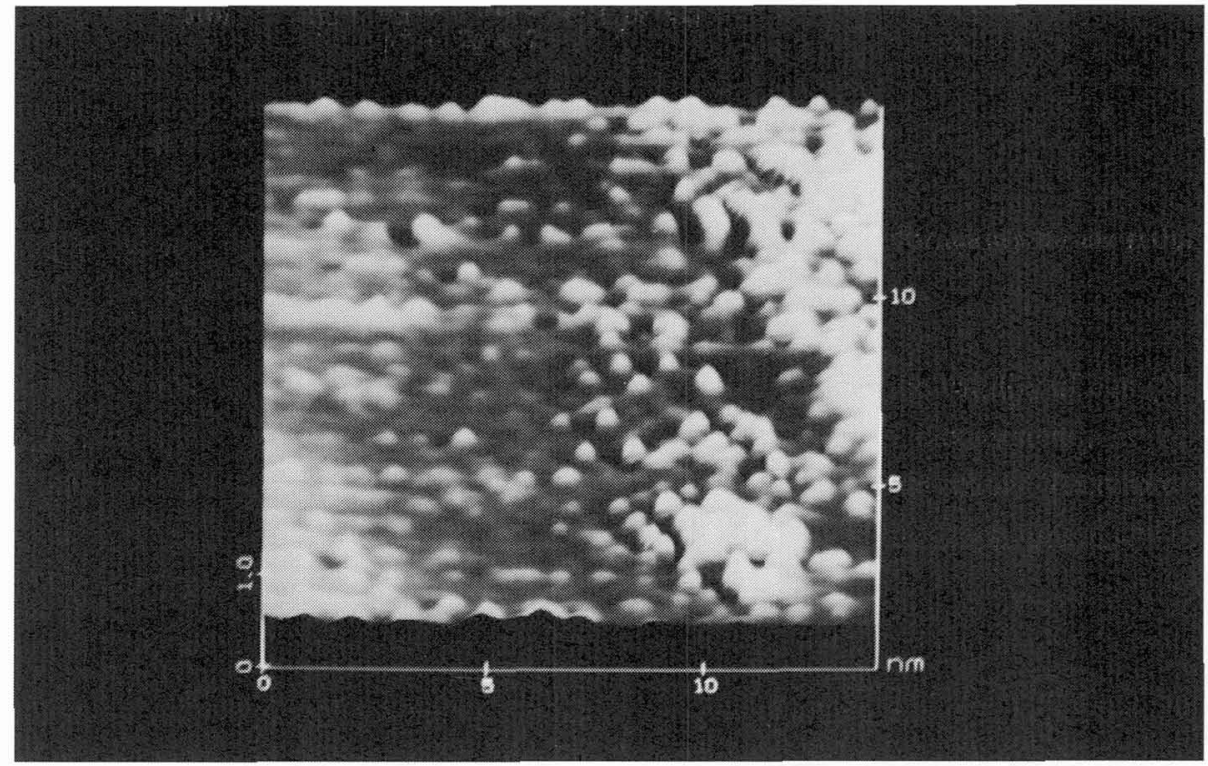

Figure $6 b$

A particularly well-ordered two dimensional array appears on the electrode surface when the potential domain is extended to comprise the third wave of $\mathrm{H}_{2} \mathrm{~W}_{12} \mathrm{O}_{40}^{6-}$ as appears on figure 7. The current output range of the potentiostat is exceeded and the current is saturated. The image of figure $7 \mathrm{a}$ has not been filtered. This same image is displayed in a larger scale in figure $7 \mathrm{~b}$ to evidence more clearly that the remarkably well-ordered domain is adjacent to thicker deposits and/or disordered areas. The molecular diameter of $10.5 \pm 0.5 \AA$ has been determined from figure $7 \mathrm{c}$, obtained by zooming in a well-ordered area of figure $7 \mathrm{~b}$ and filtered by fast-Fourier transform. The mean molecular diameter corresponds to that of Keggin type anions. It must be noticed, however, that the two-dimensional array would qualitatively resemble that of HOPG, albeit with giant entities. This observation calls for some caution in the interpretation of images even though their evolution strongly suggests that $\mathrm{H}_{2} \mathrm{~W}_{12} \mathrm{O}_{40}^{6-}$ anions are actually deposited.

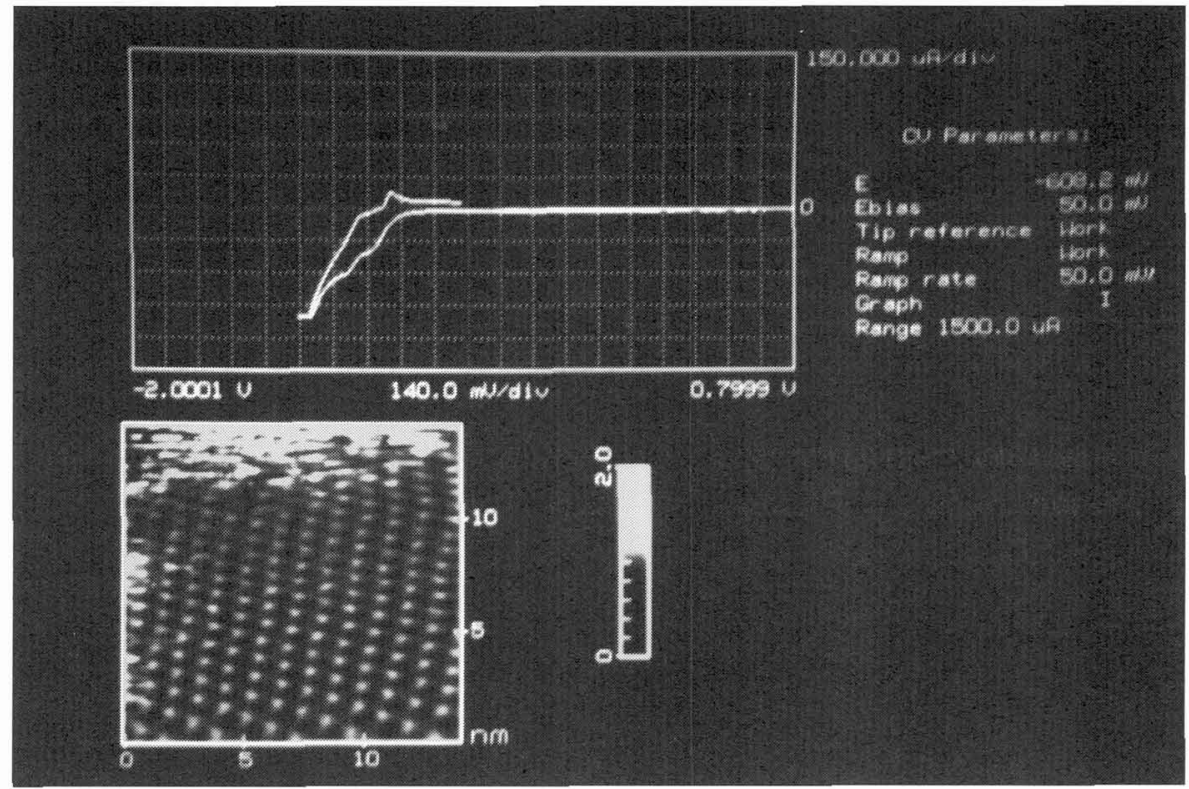

Figure $7 a$ 


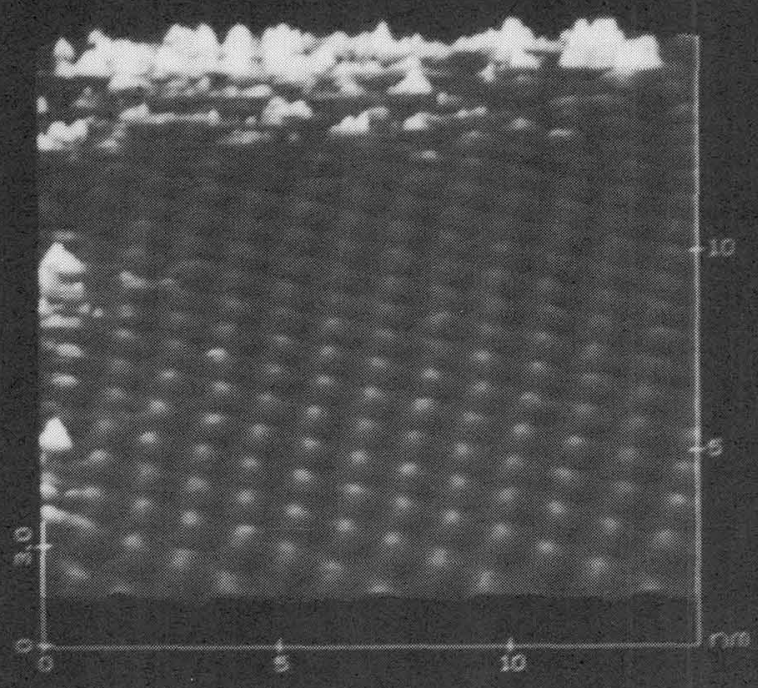

Figure $7 b$

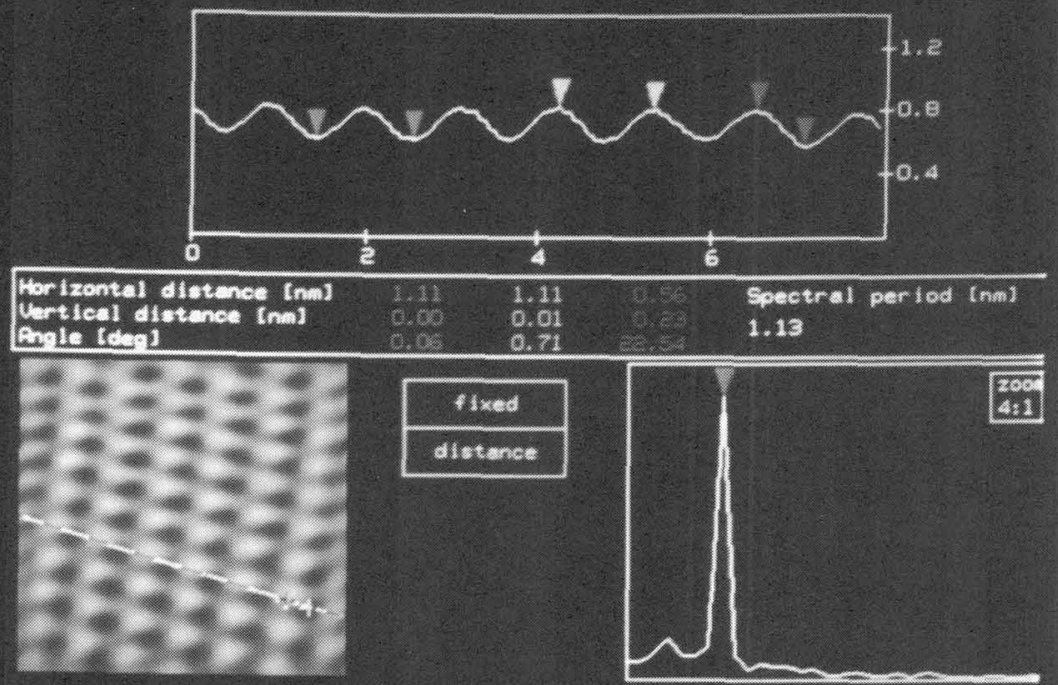

Figure $7 c$ 


\section{Concluding remarks}

The cyclic voltammograms are stable and can be cycled for very long periods of time. In each potential domain, the corresponding STM images are also reproducible. The adsorption of $\mathrm{H}_{2} \mathrm{~W}_{12} \mathrm{O}_{40}^{6-}$ on the electrode surface appears to depend on the applied potential. In the present work, the potential domain has been chosen so that completely "irreversible" adsorption of the molecules on the electrode surface does not occur. Previous work from this laboratory has demonstrated the irreversible electrochemical deposition of heteropoly or isopoly anion based catalysts on electrode surfaces at potentials more cathodic than used here [1-4]. Thus, it has been possible to monitor the evolution of the surface morphology of the working electrode during the first reduction steps of an oxometalate.

Work is in progress with STM and AFM techniques coupled in-situ with electrochemistry to obtain qualitative and quantitative informations on the oxometalate-based materials.

\section{References:}

[1] B. Keita and L. Nadjo, J. Electroanal. Chem. 191 (1985) 441

[2] B. Keita and L. Nadjo, Materials Chemistry and Physics, 22 (1989) 77

[3] B. Keita and L. Nadjo, J. Electroanal. Chem. 243 (1988) 87

[4] B. Keita and L. Nadjo, J. Electroanal. Chem. 247 (1988) 157

[5] B. Keita and L. Nadjo, Surf. Sci. 254 (1991) L 443

[6] B. Keita, K. Kjoller and L. Nadjo, Surf. Sci 256 (1991) L613

[7] B. Keita, F. Chauveau, F. Theobald, D. Bélanger and L. Nadjo, Surf. Sci. 264 (1992) 271

[8] B. Keita, K. Kjoller, E. Paris and L. Nadjo, L'Actualité Chimique : mars-avril 1992

[9] B. Keita and L. Nadjo, J. Electroanal. Chem. (in press : JEC 2830)

[10] B. Keita and L. Nadjo, J. Electroanal. Chem. 227 (1987) 77 and references there in

[11] B. Keita and L. Nadjo, G. Krier and J.F. Muller, J. Electroanal. Chem. 223 (1987) 287

[12] R. Sonnenfeld and P.K. Hansma, Science 232 (1986) 211

[13] R. Sonnenfeld and B.C. Schardt, Appl. Phys. Lett. 49 (1986) 1172

[14] A.A. Gewirth and A.J. Bard, J. Phys. Chem. 92 (1988) 5563

[15] B.A. Watson, M.A. Barteau, L. Haggerty, A.M. Lenhoff and R.S. Weber, Langmuir 8 (1992) 145. 